\title{
RELIGIÖSE PRAKTIKEN IN DER KONFESSIONELLEN HEIMERZIEHUNG UND IHRE LEGITIMATIONEN
}

Daniela Reimer

Zürcher Hochschule für angewandte Wissenschaften (ZHAW)

E-Mail:daniela.reimer@zhaw.ch

URL: https://www.zhaw.ch/de/ueber-uns/person/remr/

Zitationsvorschlag:

Reimer, Daniela (2020): Religiöse Praktiken in der konfessionellen Heimerziehung und ihre Legitimationen In: Gesellschaft - Individuum - Sozialisation (GISo). Zeitschrift für Sozialisationsforschung 1 (1). DOI: 10.26043/GISo.2020.1.4

Link zum Artikel:

https://doi.org/10.26043/GISo.2020.1.4

Dieses Werk ist lizenziert unter einer Creative Commons Namensnennung - Weitergabe unter gleichen Bedingungen 4.0 International Lizenz. 


\title{
RELIGIÖSE PRAKTIKEN IN DER KONFESSIONELLEN HEIMERZIEHUNG UND IHRE LEGITIMATIONEN
}

\author{
Daniela Reimer
}

\begin{abstract}
Im vorliegenden Text wird eine explorative Studie zu christlich-religiösen Praktiken im Alltag der konfessionellen Heimerziehung vorgestellt. In der Pilotstudie wurde in einer ausgewählten Heimgruppe methodisch mit teilnehmender Beobachtung und halboffenen Interviews mit verschiedenen Zielgruppen gearbeitet. Bei der Darstellung der Ergebnisse werden einerseits beobachtete religiöse Praktiken und Praktiken, von denen Kinder in Interviews berichten, aufgezeigt, andererseits die Legitimationsmuster der Leitungs- und Fachkräfte für diese Praktiken. Abschliessend werden Themen herausgearbeitet, die es in der weiteren Forschung zu vertiefen gilt.
\end{abstract}

Keywords: Heimerziehung, religiöse Praktiken, teilnehmende Beobachtung, Expert*inneninterviews

\section{EINLEITUNG}

Die Heimerziehung in Deutschland hat sich in den letzten Jahrzehnten stark gewandelt (Kappeler 2018; Reimer 2018). Seit der Heimkampagne der 1968er-Jahre, verbunden mit den anschliessenden Ausdifferenzierungs- und Deinstitutionalisierungsprozessen (Freigang/Wolf 2001) gelten Heimeinrichtungen nicht mehr als Disziplinierungsinstitutionen, sondern weitgehend als Orte, an denen ein gutes, kindgerechtes Aufwachsen möglich ist, wenn Aufwachsen bei einem Elternteil oder beiden Eltern vorübergehend oder längerfristig nicht möglich ist. Gleichwohl wird in aktuellen Studien deutlich, dass die Heimerziehungslandschaft aktuell höchst divers ist (in Ausgestaltung, Trägerstruktur, Qualität, Quelle) und dass es bis heute weit verbreitet Praxen in der Heimerziehung gibt, die dem guten Aufwachsen von Kindern und Jugendlichen entgegenstehen (vgl. Clark 2018, Schrödter 2018).

Rund 25\% der Kinder, Jugendlichen und jungen Erwachsenen, die in der Heimerziehung in Deutschland leben, sind in Einrichtungen mit religiöser Trägerschaft untergebracht (Destatis 2018), die meisten dieser Einrichtungen sind in evangelischer oder katholischer Trägerschaft. ${ }^{1}$ Religiöse Träger nehmen ergo quantitativ eine wichtige Rolle in der Heimerziehungslandschaft ein. Die Repräsentation religiöser Träger in der
Heimerziehung ist nur vor dem Hintergrund der deutschen Wohlfahrtstaatsentwicklung und den Strukturen der Wohlfahrtserbringung (Ehlke et al. 2017) zu verstehen. Wie die konfessionellen Träger ihre Angebote ausgestalten ist hochdivers (ebd.); es sind regionale trägerabhängige Unterschiede vorhanden, die die diverse Angebotslandschaft prägen (ebd.). Über den Lebensalltag von Kindern in konfessionellen Einrichtungen und religiöse Praktiken bei diesen Trägern im Einrichtungsalltag ist wenig bekannt, nur, dass sich statistisch die Gruppe der Kinder fast immer multireligiös zusammensetzt (Destatis 2018) und dass konfessionelle Träger glaubensgemeinschaftliche Profile aufweisen, die „nach aussen hin sicht- wie auch nach innen hin erfahrbar" (Ehlke et al. 2017: 240) sind.

Die Wohlfahrtserbringung ist also von konfessionellen Trägern durchzogen (ebd.); in der Sozialpädagogik werden die religiösen Wurzeln der Disziplin anerkannt (z.B. Oelkers 1992), allerdings ist pädagogisch sehr umstritten, ob überhaupt - und wenn ja, welche Art - religiöse Praktiken für ein gutes Aufwachsen von Kindern förderlich sind. In der (protestantischen) Religionspädagogik wird dagegen davon ausgegangen, dass "Kinder ein Recht auf Religion“ (Schweitzer 2013) haben, und es wird betont, dass Kinder religiöse Differenzen intensiv wahrnehmen (Edelbrock et al. 2010) und sich

\footnotetext{
1 Zu muslimischen Trägern in der Kinder- und Jugendhilfe in Deutschland wird derzeit intensiv geforscht, vgl. Selent et al. (2018).
} 
entwicklungspsychologisch mit religiösen Fragen auseinandersetzen (Fowler 1991; Hart 2003). Auch in der Jugendforschung wird regelmässig über den Stellenwert von Religion und religiösen Praktiken in der Lebenswirklichkeit von Kindern und Jugendlichen diskutiert (z.B. Maschke et al. 2013, S. 192 ff.).

Ein Blick auf die religiösen Praktiken im Alltag in Heimeinrichtungen und die Legitimation dieser Praktiken durch Gruppenfachkräfte und Leitungspersonal scheint entsprechend vielversprechend, weil er Einblicke verleiht einerseits in den Gruppenalltag, andererseits in die Legitimationsmuster pädagogischer Fach- und Leitungskräfte für das Vorhandensein der Praktiken. Dies ist brisant, da es sich bei der Heimerziehung um Erziehung im öffentlichen Auftrag handelt, und der Staat, der diesen Auftrag erteilt, ein säkularisierter ist. Religiöse Praktiken erscheinen vor diesem Hintergrund entsprechend begründungsbedürftig. Im Rahmen einer Pilotstudie wurde 2017/18 in einer Heimgruppe eines katholischen Trägers in Deutschland mit teilnehmender Beobachtung, Interviews mit Kindern aus der Gruppe und Expert*inneninterviews mit Gruppenerzieher*innen und der Heimleitung ein erster Zugang für eine Erforschung des skizzierten Phänomens erprobt. Es handelt sich folglich um eine Einzelfallstudie. Es ist davon auszugehen, dass einige Fragen, die durch die Studie aufgeworfen werden können, sich in Variationen auch in anderen ähnlichen Kontexten stellen und daher für die Jugendhilfe im Allgemeinen von Interesse sind.

Im Folgenden werde ich kurz erläutern, was ich unter religiösen Praktiken im vorliegenden Kontext verstehe. Dann folgen einige Anmerkungen zur Verbindung von christlich-religiösen Trägern und Heimerziehung vorangestellt. Im nächsten Schritt wird die skizzierte Pilotstudie vorgestellt: Es werden die gewählten Methoden - teilnehmende Beobachtung und offene Interviews - sowie erste Analysen präsentiert. Abschliessend werden die ersten Ergebnisse in grösseren Diskurszusammenhängen verortet und es wird der weitere Forschungsbedarf skizziert.

\section{RELIGIÖSE PRAKTIKEN IM ALLTAG}

Die Studie, auf der dieser Artikel basiert, orientiert sich an dem Religionsbegriff der Heimgruppe, in der die Untersuchung durchgeführt wurde. Dabei handelt es sich um einen substantialistischen Religionsbegriff, der auf den kollektiv definierten Transzendenzglauben an eine höhere Macht verweist, die ganz im christlichen Sinne als "Gott“ bezeichnet wird. Als religiöse Praktiken in der Heimeinrichtung werden Symbole, Handlungen und Interaktionen bezeichnet, über die ein in der Einrichtung und Heimgruppe geteiltes Wissen existiert, dass sie auf eine göttliche Macht oder auf Glaubensvorstellungen verweisen (z.B. Kreuzsymbol, Nonnentracht) oder dazu dienen, mit dieser göttlichen Macht in Kontakt zu treten (z.B. Beten, Pilgern, Fasten). Ausserdem umfassen religiöse Praktiken solche, über die implizit oder explizit religiös fundierte Werte- und Moralvorstellungen vermittelt werden (z.B. man darf nicht lügen, weil das in den zehn Geboten steht), und die sich in pädagogischen Überzeugungen, Einrichtungskonzepten sowie daraus resultierend Interaktionen mit den Kindern und Jugendlichen wiederfinden (z.B. caritative Orientierung, konzeptionell verankerte Sexualmoral). Religiöse Praktiken, die beobachtet wurden und von denen mir in Interviews oder während meiner Anwesenheit in der Gruppe berichtet wurde, werden in der Analyse auch auf ihre Funktionen hin untersucht, die sie für die Heimgruppe, die Einrichtung und die Beteiligten haben.

\section{CHRISTLICHE RELIGION UND HEIMERZIE- HUNG, HISTORISCH UND AKTUELL - AN- MERKUNGEN}

Die Sorge um Witwen und Waisen ist im Judentum wie auch im Christentum ein Auftrag an die Gläubigen, genauso wie das Doppelgebot der Gottes- und der Nächstenliebe. Bereits in den Anfängen des Christentums, in einer Zeit, in der nicht erwünschte und nicht den Normalitätsvorstellungen der Gesellschaft entsprechende Kinder in grosser Zahl umgebracht wurden (Niederberger 1997), unterhielten Klöster und kirchliche Einrichtungen Babyklappen, Findelhäuser, Spitäler, Klosterschulen und Armenhäuser. Das kirchliche Unterstützungsangebot galt prinzipiell allen Kindern, um die sich leibliche Eltern nicht kümmern konnten oder wollten. Die Motivation, Kinder caritativ zu unterstützen, war nicht kindorientiert oder mildtätig, sondern galt den Gläubigen als Pflicht für das eigene 
Seelenheil. Niederberger zitiert aus einer mittelalterlichen Schrift:

Damit wir niemandem zur Last fallen oder nicht selber eine Sünde begehen, ist uns beigebracht worden, dass es sündhaft ist, Kinder, auch neugeborene, auszusetzen, und zwar vorwiegend, weil wir erleben, dass fast alle, die ausgesetzt wurden (nicht nur Mädchen, auch Knaben), zur Prostitution erzogen wurden. (ebd.: 25)

Auch der Protestantismus hat seit seinen Beginnen Anstalten und Armenfürsorge betrieben. Besonders bekannte Beispiele sind die pietistisch geprägten halleschen Anstalten von August Hermann Francke, bei denen im Zentrum stand, die Kinder zu Fleiss, Wahrheit und Gehorsam zu erziehen. In der katholischen wie in der protestantischen Anstaltserziehung standen bis in die Mitte des 20. Jahrhunderts die Anliegen der Gesellschaft, Normierung und Erziehung zur Arbeitsamkeit, und nicht die Anliegen des individuellen Kindes im Vordergrund. Auch im modernen Wohlfahrtsstaat werden viele Leistungen, auch im Bereich der Heimerziehung, von kirchlichen Trägern erbracht (Ehlke et al. 2017). Die Kritik der 1968er-Bewegung an der Heimerziehung und deren Ausrichtung auf gesellschaftliche Bedürfnisse und nicht auf die Bedürfnisse des Kindes (vgl. Reimer 2018; Kappler 2018) hat jedoch ein sehr kritisches Licht auf die Zustände in kirchlichen Einrichtungen in den 1950er- und 1960er-Jahren geworfen: Kinder, die zum Gebet und zur Arbeit gezwungen wurden, straftheologisch geprägte pädagogische Vorstellungen, schlecht oder gar nicht qualifiziertes Personal, Körperstrafen, sexuelle und psychische Gewalt sind nur einige Stichworte (vgl. AGJ 2010). Viele kirchliche Einrichtungen im deutschsprachigen Raum haben - nicht ohne Druck - in den letzten beiden Jahrzehnten ihre Geschichte aufgearbeitet (vgl. Wensierski 2006; AGJ 2010). An die Stelle gesellschaftlich orientierter Erziehungsanliegen sind mittlerweile in der Kinder- und Jugendhilfe zumindest strukturell und im Kinderund Jugendhilfegesetz $(\mathrm{KJHG})$ verankert die individuellen Bedürfnisse gerückt: das Kindeswohl, Lebensweltorientierung (Thiersch 2014), Entstigmatisierung v.a. durch Dezentralisierung (Freigang/Wolf 2001) und an vielen Orten Familialisierung (Richter 2013; Wolf 2005) stehen im Zentrum. Auch sind seit den 1990er-Jahren klare Grenzen zwischen Unterbringungen in Pflegefamilien und Heimeinrichtungen verschwommen (Freigang/Wolf 2001) und verschwimmen weiter (Wolf 2005). Diese Transformationen haben auch kirchliche Einrichtungen verändert. Während in den letzten Jahren viel Wissen generiert wurde über religiöse Sozialisation in den Heimen der Nachkriegszeit (AGJ 2010), ist weitgehend unbekannt, welchen Stellenwert religiöse Praktiken in Heimen kirchlicher Träger heute haben, obwohl davon ausgegangen werden kann, dass religiöse Praktiken auch heute in der konfessionellen Heimerziehung zu finden sind - wie durch die gesamte Geschichte der christlich geprägten Heimerziehung. ${ }^{2}$ Dieses Desiderat ist aufgrund des hohen Anteils der konfessionellen Einrichtungen erstaunlich (Destatis 2018), auch ist es bemerkenswert vor dem Hintergrund, dass in den letzten Jahren zunehmend ethnografische Forschungen im Heimerziehungsbereich zu einer grossen Breite an Themen entstanden sind, wie zum Beispiel Emotionen (Schröder 2017), Strafen (Schrödter 2018), Essenssituationen (Behnisch 2018; Rose/Adio-Zimmermann 2018), Schule/Bildung (Kliche/Täubig 2016), Doing Familiy (Eßer 2013) - der Bereich der religiösen Praktiken fand dabei aber bisher kaum Beachtung.

\section{FELDZUGANG UND FORSCHUNGSME- THODEN}

Um diesem Forschungsdesiderat nachzugehen, habe ich im Winter 2017 eine kleine, auf eine Heimgruppe in einer katholischen Einrichtung fokussierte explorative Studie gestartet. Da ich selbst weder Forschungs- noch eigene sozialpädagogische Berufserfahrung im Feld der Heimerziehung habe, war für mich als Erziehungswissenschaftlerin und Nichtkatholikin nicht nur

\footnotetext{
2 Eine aktuelle und umfangreiche Studie, die mit Interviews und Beobachtungen arbeitet, gibt es lediglich zur Situation in der Schweiz (Schallberger/Schwendener 2017). Allerdings ist diese Studie auf den bundesdeutschen Kontext, aufgrund der sich unterscheidenden Strukturen der Jugendhilfe in Deutschland und der Schweiz und dem Fokus auf freikirchliche/evangelikal geprägte Heimeinrichtungsträger, die es in Deutschland kaum gibt und deren Religiosität sich deutlich von der der Landeskirchen unterscheidet, nur bedingt übertragbar.
} 
das Thema Religion und Katholizismus, sondern auch das Thema Heimerziehung neu. Im Rahmen der explorativen Pilotstudie habe ich mich entschieden, einen multimethodischen Ansatz zu nutzen, bei dem die Thematik exploriert werden kann. Im Folgenden werden die verschiedenen Forschungszugänge beschrieben.

\subsection{Informelle Gespräche mit Kolleg*innen}

Informelle Gespräche mit Fachkolleg*innen, die in Einrichtungen religiöser Träger tätig sind oder waren, waren für mich ein erster Ansatz, um Informationen zum Feld, zu den Logiken des Feldes und zu Zugangsmöglichkeiten zu bekommen. Auch wenn diese Vorarbeit nur bedingt als forschungsmethodischer Hintergrund bezeichnet werden kann, gilt diese als wichtiger Teil der explorativen Studie. In den Gesprächen wurde mir vermittelt, dass es schwierig werden würde, im Rahmen einer teilnehmenden Beobachtung religiöse Praktiken zu beobachten, da diese nur einen sehr geringen Anteil des Alltagsgeschehens in Heimgruppen ausmachen. Mehrere Fachkolleg*innen betonten, dass die Tätigkeit in einer Einrichtung mit religiöser Trägerschaft sich nicht von der Arbeit in einer Einrichtung mit einer anderen Trägerschaft unterscheide. $^{3}$ In diversen Gesprächen konnte ich erarbeiten, dass sich die Vorweihnachtszeit in besonderer Weise eignen könnte, um religiöse Praktiken in einer Heimgruppe zu beobachten. Entsprechend wählte ich für die Pilotstudie diese Jahreszeit aus.

4.2 Teilnehmende Beobachtung in einer Heimgruppe

Durch die Vermittlung eines Fachkollegen ist es gelungen, Zugang zu einer katholischen Heimeinrichtung zu erhalten. Die Heimeinrichtung verfügt über ein Haupthaus, auch Mutterhaus genannt, in dem sich ein Kloster befindet, mehrere Heimgruppen sowie administrative Räumlichkeiten. Darüber hinaus gibt es diverse Aussenwohngruppen. Der Kinder- und Jugendhilfebereich ist ein Teilbereich des Trägers. Die Heimgruppe, in der die Beobachtung stattfand, ist eine Aussenwohngruppe der Einrichtung. Sie befindet sich in einem Einfamilienhaus in $20 \mathrm{~km}$ Distanz zum Haupthaus. Sie ist dörflich gelegen, umringt von der Ortskirche, dem Pfarr- und Gemeindehaus sowie dem Friedhof. In der Heimgruppe leben acht Jungen und Mädchen zwischen 8 und 15 Jahren, sie werden von diversen Erzieher*innen, einer Hauswirtschaftskraft und einem Praktikanten im Schichtdienst betreut. Die Kinder leben unterschiedlich lange in der Heimgruppe, die meisten haben vor der Platzierung in der Gruppe andere Heim- oder Pflegefamiliensettings durchlaufen. Sie kommen überwiegend aus Familien, in denen Religion keine oder eine untergeordnete Rolle spielt, zwei Jungen - Brüder - stammen aus einer muslimischen Familie; es ist bekannt, dass die Eltern Muslime sind, inwiefern die Religion für die Herkunftseltern eine Rolle spielt, wird von den Erzieher*innen unterschiedlich eingeschätzt.

Ich konnte ein erstes Telefonat mit der Gruppenleitung führen, die Räumlichkeiten und Erzieher*innen danach in einem Gespräch mit der Gruppenleitung kennenlernen und die Heimgruppe in der Folge zu fünf Terminen besuchen, mich wie eine „faule Praktikantin“ (Wolf 1999) im Gruppenalltag verhalten und Beobachtungen sammeln; dabei wurde changiert zwischen der Rolle der beobachtenden Teilnehmerin und der teilnehmenden Beobachterin (vgl. Kliche/Täubig 2016). Die Dokumentation erfolgte in Form von Gedächtnisprotokollen jeweils im direkten Anschluss an die Termine. Die Besuche hatten verschiedene Schwerpunkte: Zwei fanden im Gruppenalltag an einem Nachmittag innerhalb der Woche statt, einer an einem Samstag, inkl. Weihnachtsmarktbesuch; bei einem Beobachtungsbesuch konnte ich mit einer Erzieherin und der Gruppe an einer Weihnachtsfeier im Haupthaus teilnehmen und ein Besuch fand an einem Sonntagnachmittag statt. Aufgrund des explorativen Charakters der Studie war die Beobachtung nicht hypothesengeleitet, sondern offen (vgl. Merkens 1992), gleichwohl mit einem starken Fokus auf die religiösen Praktiken im Gruppenalltag. Dabei habe ich nicht nur die Räume, Handlungen und Interaktionen von Erzieher*innen und Kindern beobachtet, sondern auch mich selbst und die Wirkung der Praktiken auf mich. Direkt

\footnotetext{
${ }^{3}$ Abgesehen von Einstellungsvoraussetzungen (in der Regel Zugehörigkeit zu einer Kirche) und Einschränkungen im Streikrecht.
} 
nach den Beobachtungsterminen wurden jeweils sehr ausführliche schriftliche Protokolle erstellt, in denen viele einzelne Szenen und Eindrücke beschrieben wurden. Diese wurden gespeichert, in mehreren Überarbeitungsschritten in der Textform immer weiter verdichtet und es wurden erste Hypothesen formuliert. Im Verlauf der weiteren Beobachtung wurden weitere Hypothesen generiert, bei den Folgeterminen jeweils modifiziert und in den Überarbeitungen der Protokolle zunehmend zu gemeinsamen Hypothesen verdichtet. Die Beobachtungsprotokolle und ihre Be- und Überarbeitungen beschreiben daher auch einen Prozess der Hypothesenverdichtung (ebd.). Beobachtungen, Protokollierungen und Analyse stehen entsprechend in einem fliessenden Zusammenhang.

4.3 Interviews mit Gruppenleitung, Erzieherin, Praktikant

Am Ende der Beobachtungsphase habe ich leitfadengestützte Expert*inneninterviews (vgl. Helfferich 2014) mit der Gruppenleiterin, einer Erzieherin der Gruppe sowie dem Praktikanten der Gruppe geführt. Der Leitfaden umfasste ein Set von Fragen, die sich um religiöse Praktiken im Gruppenalltag, die institutionelle Verankerung der Einrichtung und mögliche daraus hervorgehende Erwartungen an das Personal, aber auch den persönlichen Bezug der Befragten zu religiösen Themen, drehen. Die Interviews fanden alle in der Heimgruppe statt, in einem abgetrennten Zimmer. Sie dauerten zwischen 30 und 60 Minuten. Die verschiedenen Interviewten stehen dabei auch für verschiedene Positionen: Die Gruppenleitung ist für die Gesamtgruppe verantwortlich, auch konzeptionell. Die befragte Erzieherin ist Mitglied im sogenannten Arbeitskreis Seelsorge, dem aus jeder Heimgruppe der Einrichtung eine Fachkraft angehört. Ihre Aufgabe ist es, das Thema Religion auf der Ebene des Teams der Heimgruppe einzubringen und Anregungen aus dem Arbeitskreis umzusetzen. Der Praktikant war während meiner Beobachtungsphase und zum Zeitpunkt des Interviews erst wenige Monate in der Gruppe beschäftigt. Er hatte damit eine Aussenperspektive und ist ausser der Hauswirtschaftskraft der einzige Erwachsene in der Heimgruppe, der über keine pädagogische Ausbildung verfügt.

\subsection{Interview mit der Einrichtungsleitung}

Um die Einrichtungsperspektive zu erheben habe ich ausserdem, mit einem leicht modifizierten Leitfaden, ein Expert*inneninterview mit der Leitung der Kinder- und Jugendhilfeabteilung der Einrichtung geführt. Das Interview fand im sogenannten Mutterhaus statt. Interviewt wurden gemeinsam der Leiter der Heimeinrichtung und die sogenannte Seelsorgebeauftragte. Der Einrichtungsleiter ist Sozialpädagoge, die Seelsorgebeauftragte Religionspädagogin. Das Interview dauerte ca. 90 Minuten, die beiden Interviewten hatten gleiche Gesprächsanteile.

\subsection{Interviews mit zwei Kindern}

Angelehnt an die Methode der theologischen Gesprächsführung mit Kindern (FreudenbergerLotz 2012) konnte ich ausserdem mit zwei Mädchen aus der Heimgruppe Interviews führen. Die Methode zentriert Gottesvorstellungen und religiöse Praktiken im Alltag der Kinder. So werden Kinder aufgefordert, ihre Vorstellung von Gott kreativ in einem Bild umzusetzen und das Gemalte zu erläutern, ausserdem werden Fragen zu religiösen Praktiken und deren Relevanz im Alltag der Kinder gestellt. Die beiden Mädchen hatten sich freiwillig für Interviews bereiterklärt, von den Erzieher*innen waren sie mir als besonders interessiert an christlich-religiösen Fragen beschrieben worden. Die Interviews fanden in den Zimmern der Mädchen in der Heimgruppe statt. In den konkreten Gesprächssituationen gibt es viele Redepausen, auf die Aufforderung zur kreativen Gestaltung am Anfang des Interviews („malen, wie ich mir Gott vorstelle“) konnte sich nur ein Mädchen einlassen. Beide Interviews verliefen sehr emotional - ein Mädchen hat im Interview viel geweint, als sie über den Tod ihres Vaters und den Trauerprozess erzählt hat. Die beiden Interviews dauerten jeweils zwischen 30 und 60 Minuten.

\subsection{Interviewanalyse}

Alle Interviews wurden vollständig transkribiert. Die Expert*inneninterviews wurden anknüpfend an das themenzentriert-komparative Verfahren (Lenz 1986) mit MAXQDA induktiv codiert, d.h. Kategorien wurden im Verlauf des Codierungsprozesses gebildet und die Codes so lange 
umbenannt, bis sich im Vergleich der Interviews Codes bildeten, nach denen alle Interviews codierbar waren. Im Verlauf wurden grosse Kategorien gebildet und Unterkategorien. In einem nächsten Auswertungsschritt wurden alle Codes daraufhin analysiert, inwiefern sie Legitimationen für die religiösen Praktiken in der Einrichtung/in der Gruppe darstellen. Daraus konnten sieben Legitimationsmuster herausgearbeitet werden. Anhand dieser wurden die Interviews abschliessend nochmals codiert und aus den Codes Substrate erstellt.

Die Interviews mit den Kindern wurden ebenfalls in MAXQDA eingepflegt, sie wurden im ersten Schritt mit ausführlichen Memos versehen; im Anschluss wurden drei grosse Themen herausgearbeitet und die Interviews wurden damit codiert.

\section{ANALYSE UND ERGEBNISSE}

\subsection{Religiöse Praktiken im Alltag}

Aus den teilnehmenden Beobachtungen konnten drei Hypothesen verdichtet werden. Die ersten beiden Hypothesen stehen dabei in einem engen Zusammenhang, beleuchten aber verschiedene Facetten desselben Aspekts. Die dritte Hypothese beleuchtet einen anderen Aspekt.

Religiöse Praktiken durchziehen den Alltag ohne dass es den Fachkräften bewusst ist

Durch die Beobachtungen zieht sich ein Widerspruch, der den Fachkräften im Laufe meiner Anwesenheit bewusst und auch zunehmend von innen verbalisiert wurde. Die Fachkräfte und vor allem die Gruppenleitung halten sich selbst und die Gruppe für nicht besonders religiös. In unserem Auftakttelefonat äusserte die Gruppenleiterin mehrfach, dass die Gruppe nicht religiös sei und es daher für mich wenig zu beobachten gäbe. Bereits im ersten Gespräch revidierte sie diesen Eindruck. Im Protokoll habe ich vermerkt:

Frau Meier berichtet mir, dass sie - auch im Gespräch mit den Kolleg*innen - festgestellt habe, dass der Bezug zu Religion und Glaube in der Gruppe doch recht intensiv sei. Lachend stellt sie fest, dass sie räumlich von kirchlichen Gebäuden und Grundstücken umgebene sind, Kirche, Pfarrhaus, Gemeindehaus, Friedhof [...]. Da die Gruppe zwischen der Kirche und dem Friedhof liegt, führt der Zug mit dem Sarg regelmäßig bei Beerdigungen am Haus vorbei, die Kinder schauen dann immer gerne zu, vor allem neue Kinder; das führt zu vielerlei Gesprächen über Tod und Glaube. [...] Weiter erklärt sie, dass sie beim Nachdenken festgestellt habe, dass Kirche und Glaube für die Kinder wichtig sei. Sie betont mehrfach, dass die Kinder dort Zugehörigkeit erleben und finden. Ein Kind habe sich im Alter von acht Jahren in der naheliegenden Kirche taufen lassen. Die Herkunftseltern, die nicht gläubig/religiös sind haben teilgenommen und mitgefeiert. Ein anderes Mädchen hat kürzlich (im Sommer) ihren Vater verloren. Sie geht regelmäßig zur katholischen Kirche, um eine Kerze für den verstorbenen Vater anzuzünden. Außerdem haben dieses Mädchen und ein weiterer Junge beim Pilgerangebot der Einrichtung im vergangenen Sommer teilgenommen, was eine sehr positive Erfahrung gewesen sei. Ins Gespräch schaltet sich kurzzeitig die Haushaltshilfe, die in der angrenzenden Küche kocht, ein. Sie berichtet, dass die Einrichtung viele spirituelle Angebote für die Mitarbeiter*innen bereithält, die sie, aber auch die anderen Kolleginnen der Gruppe, regelmäßig annehmen. So gebe es regelmäßige Gottesdienste, Stille Tage, ein Highlight sei eine gemeinsame Pilgerreise der Mitarbeiter*innen nach Assisi gewesen.

In den wenigen Protokollzeilen wird deutlich, dass der Alltag für Kinder wie Mitarbeiter*innen der Gruppe von religiösen Praktiken durchzogen ist und dies der Gruppenleiterin erst durch eine Reflexion über meine Fragestellung und anknüpfende Gespräche mit ihren Kolleg*innen zugänglich geworden ist. Es werden sowohl räumliche Elemente, kommunikative Elemente aber auch freizeit- und lebensstrukturierende Elemente benannt, die von religiösen Praktiken durchzogen sind.

Tatsächlich konnte ich bereits bei der Raumgestaltung viele religiöse Symbole entdecken: Kreuz an der Wand, Bild der Ordensfrau, die die Einrichtung gegründet hat, christliche Bücher im Bücherregal, Weihnachtsdekoration, Adventskalender, ein christliches Buch als literarischer Adventskalender und in bzw. vor den Zimmern 
der Kinder Fotos von Taufen, Kommunionen und Konfirmationen. Auch im Gruppenalltag sind religiöse Praktiken in den Alltagsroutinen ständig präsent: Vor dem Essen wird gebetet, inkl. Bekreuzigen vor und nach dem Gebet. Gebet und Bekreuzigen sind zwar, wie mir erklärt wurde, freiwillig, es machen aber alle mit, auch die Kinder mit muslimischen Familienhintergrund. Auch ich konnte an mir beobachten, wie ich versuchte mitzumachen. Ausserdem gab es im von mir beobachteten Zeitraum regelmässiges Vorlesen christlicher Bücher, Gespräche über Weihnachten und Weihnachtsgeschenke, bei denen auf den christlichen Hintergrund des Festes verwiesen wurde, ein Gottesdienst mit Weihnachtsfeier im Mutterhaus mit den Nonnen, der zwar ein freiwilliges Angebot war, bei dem aber alle Kinder der Gruppe teilnahmen sowie einen Weihnachtsmarktbesuch, bei dem wir - eine Erzieherin, zwei Kinder der Gruppe und ich - lange bei einem Stand mit Krippenfiguren verweilten, das Christuskind suchten und die Erzieherin die Weihnachtsgeschichte erzählt hat. Und es gab Gespräche über den Zweck der Sternsinger und über die Möglichkeit, bei den Sternsingern der örtlichen katholischen Gemeinde mitzumachen, und es gab Berichte über andere Aktivitäten, denen die Kinder im Rahmen der Kirchengemeinde nachgehen. Kinder aus der Gruppe erzählten von den Pilgerangeboten der Einrichtung und vom vorösterlichen Fasten in der Gruppe und Erzieher*innen berichteten von der Teilnahme an den "Stillen Tagen" und anderen Seelsorgeangeboten und ihren Erfahrungen damit. Bewusste Versuche von Kindern, sich religiösen Praktiken zu entziehen, konnte ich in keiner Situation beobachten. Die Kinder nahmen religiöse Angebote mit grosser Offenheit an, diskutierten wer das Tischgebet sprechen darf, nahmen freiwillig an der Weihnachtsfeier mit den Nonnen und am Gottesdienst teil, wollten gerne Sternsinger sein und planten bereits in der Adventszeit die Fastenzeit. Über das Pilgern haben sie ausschliesslich positiv berichtet.

Religiöse Praktiken, so die Hypothese, sind in der Heimgruppe so präsent, dass es nicht möglich ist, sich ihnen zu entziehen. Den Erzieher*innen sind sie in ihrer Ritualisierung kaum reflexiv zugänglich. Die Kinder nehmen die religiösen Praktiken mit grosser Offenheit als Teil ihrer Lebensund Alltagswelt in der Heimgruppe an.
Religiöse Praktiken sind in der Gruppe selbstverständlich und unhinterfragt

Eng damit verbunden ist, so die zweite Hypothese, dass in der Gruppe trotz der interreligiösen Zusammensetzung kein interreligiöser Ansatz gepflegt wird, sondern explizit katholisch geprägte religiöse Praktiken selbstverständlich und unhinterfragt Teil des Alltags sind. Das zeigt sich besonders an den Symbolen in der Hausdekoration - Kreuz, Bild von Ordensfrau - sowie am Bekreuzigen vor und nach dem Tischgebet, das auch ein leibliches Kennzeichnen der Zugehörigkeit zur katholischen Glaubensgemeinschaft darstellt und über das ich im Protokoll notiert habe, dass ich selbst bei mir beobachtet habe, wie ich versucht habe, das Ritual - genauso wie allen anderen auch - zu vollziehen, und gleichzeitig mit der Situation überfordert war. Die Tischgebete, die meist mit einem Gebetswürfel gewürfelt werden, sind überwiegend explizit christliche und nicht solche, die allen monotheistischen Religionen zugeordnet werden können, sprich es wird explizit "Jesus“ und nicht "Gott" adressiert. Und der Gottesdienst, an dem wir teilgenommen haben, war ein explizit katholischer; zwar gab es einen kurzen Verweis darauf, dass es auch andere Religionen und Glaubensrichtungen gibt, aber es folgten keine weiteren Ausführungen dazu. Trotz der Betonung der Erzieher*innen in Gesprächen mit mir, dass andere Glaubensrichtungen wertgeschätzt werden, und obwohl stets betont wurde, dass es ja zwei „muslimische Kinder" in der Gruppe gebe, wurde die (vermeintliche) muslimische Religion für mich nicht beobachtbar thematisiert. Eine Erzieherin berichtete mir bzgl. der beiden "muslimischen Kinder“ in einem Gespräch, dass das beim Gruppeneintritt der Kinder ausgesprochene Verbot, Schweinefleisch zu konsumieren, nicht umgesetzt wird. Begründung war auf Nachfrage, dass die Kinder unterernährt in die Gruppe gekommen seien und der Verzicht auf Schweinefleisch in dieser Situation problematisch gewesen wäre.

Die Selbstverständlichkeit, mit der explizit christlich religiöse Praktiken Teil des Gruppenalltags sind, wird auch auf die Ebene der Elternarbeit ausgeweitet. Bei meinem letzten Besuch in der Heimgruppe wurde mir von der Weihnachtsfeier in der Gruppe berichtet, zu der die Eltern der Kinder eingeladen waren. Die Einladung erfolgte 
explizit als Einladung zur Weihnachtsfeier. Während die Erzieherin positiv über die Feier erzählte, berichtete mir der Praktikant, dass die Kinder, deren Eltern der Einladung nicht gefolgt waren, geweint haben und dass er sie trösten musste und die Feier als anstrengend empfunden habe. Die explizite Ausrichtung von Weihnachten als Fest der Familie sei für die Kinder der Heimgruppe problematisch, erläuterte er, da viele der Kinder darunter leiden, dass sie nicht in ihren Herkunftsfamilien leben können und die Familien dem Idealbild nicht entsprechen.

Religiöse Praktiken werden bei diversen Themen genutzt, oft normierend und moralisierend

Im Gruppenalltag werden immer wieder religiöse Praktiken herangezogen, um normierend und moralisierend auf einzelne Kinder oder das Gruppengeschehen einzuwirken, so die dritte Hypothese. Im Verlauf der Beobachtung hat sich gezeigt, dass auch die Erzieher*innen der Gruppe bestimmte religiöse Praktiken als normierend erleben. So war die Erzieherin, die ich immer als angenehm entspannt und geduldig mit den Kindern erlebt hatte, vor dem Weihnachtsgottesdienst im Haupthaus plötzlich sehr angespannt und hat die Kinder vor dem Eingang zum Mutterhaus vehement dazu angehalten, sich in den kommenden Stunden mit Verweis auf den Gottesdienst und die anwesenden Geistlichen zu benehmen. $^{4}$

Auch in anderen Situationen konnte ich die normierende Funktion wahrnehmen. Ein Auszug aus einem Protokoll, es geht um eine Essenssituation, verdeutlicht das:

Während des Essens unterhalten sich die Kinder lebhaft, ein großes Thema sind Weihnachtsgeschenke. Die 15-jährige Anna spricht von Sportschuhen, die $200 €$ kosten, und die sie sich von ihren Eltern wünscht [...]. In Richtung Anna sagt die Erzieherin mehrfach, bei Weihnachten gehe es nicht um Konsum, darüber hätten sie bereits mehrfach gesprochen und sie wolle auch heute Abend nochmals mit ihr darüber sprechen [...]. Ich setzte mich nach dem Abendessen zur Erzieherin in die Küche, sie spült und erzählt mir, dass der Konsum um Weihnachten sie sehr stört, sie selbst und ihre Familie machen sich keine Geschenke, wichtig sei es, dass man Zeit zusammen hat. Das wolle sie auch den Kindern vermitteln. Sie berichtet, [...] dass es in Spanien erst am Heilige Drei Könige kleine Geschenke gäbe und sie dies besser fände. An Weihnachten wird die Geburt Christi gefeiert, dafür bedarf es keiner Geschenke. Am 6.1. würden die Geschenke symbolisch besser passen, da die Heiligen Drei Könige dem Jesuskind Geschenke gebracht hätten.

Weihnachten ist in der Gruppe das Fest der Familie - der guten, funktionierenden, "normalen“ Familie. Das erklärt möglicherweise auch den Versuch der Erzieher*innen, Weihnachten mit den Kindern und deren Eltern gemeinsam zu feiern. Deutlich wird dies aber auch im folgenden Protokollauszug:

Ich erkundige mich bei Erzieherin Karin, ob es Kinder gibt, die Weihnachten in der Gruppe bleiben. Sie bejaht, ein Mädchen, auf eigenen Wunsch, sowie drei Jungen. Sie berichtet von der Überlegung, die Kinder am Weihnachtsfeiertag mit zu sich nach Hause zu nehmen, damit sie mal rauskämen, und normale Weihnachten in einer normalen Familie erlebten. Und nicht wie in ihren Herkunftsfamilien mit viel Streit. Einfach harmonische Weihnachten in einer normalen Familie. Sie ergänzt, sie habe schon einmal einige Kinder an Weihnachten mit zu sich nach Hause genommen und das sei sehr schön gewesen.

In beiden Protokollausschnitten wird die Normierung durch die religiösen Praktiken am Beispiel von Weihnachten deutlich. Im ersten Zitat wird die religiös fundierte Konsumkritik deutlich vorgetragen - dahinter wird der Wunsch des Mädchens nach einem Geschenk von den Eltern,

\footnotetext{
${ }^{4}$ Prinzipiell wäre vorstellbar, dass in öffentlichen Situationen von Seiten der Erzieher*innen ähnlich normierend agiert wird, allerdings konnte ich das nie beobachten, obwohl ich mich mehrfach mit Erzieher*innen und Kindern in öffentlichen Situationen befand. Dass die Normierung der Gruppe vor dem Gottesdienst durch die in dieser Situation offensichtlich selbst normierte Erzieherin erfolgte, ist insofern besonders, als dass es sich um eine interne Veranstaltung handelte, an der nur Kinder und Jugendliche aus der Einrichtung, Mitarbeitende, Leitungskräfte und die Nonnen des Klosters teilnahmen, also ein enger Rahmen, der aber möglicherweise genau deshalb als interne Situation im religiösen Kontext die massive Normierung hervorgerufen hat.
} 
vermutlich verbunden mit dem Wunsch nach Anerkennung durch die Herkunft, abgewertet und zentrale Bedürfnisse des Kindes werden übergangen. In beiden Ausschnitten wird darüber hinaus das Thema Familie normierend verhandelt. Weihnachten wird als Fest der (heiligen) Familie zelebriert, die „normale“ Familie wird zum Ideal erhoben, während die Herkunftsfamilien der Kinder als abweichend abgewertet werden.

\subsection{Kinderinterviews}

In den Interviews mit den beiden Mädchen der Gruppe verdichten sich zwei Themenbereiche: die Präsenz religiöser Praktiken in der Gruppe das bestätigen die Beobachtungen - sowie die Verortung der religiösen Praktiken zwischen Ressource und Begrenzung aus der Perspektive der Kinder.

Beide Mädchen berichten, sie hatten vor ihrem Einzug in der Heimgruppe keinen oder wenig Kontakt und Bezug zur christlichen Religion. Beide betonen, dass sie erstmals in der Heimgruppe intensiv mit religiösen Praktiken konfrontiert wurden und diese für sich als Bereicherung erleben. Gott beschreiben die Mädchen als gross, mächtig, nett und gerecht, er „beschützt auf allen Wegen" und "spielt mit dem Engel Gabriel". Beide Mädchen haben eine Vorstellung von einem persönlichen Gott, der sich um ihre Anliegen kümmert; dies spiegelt sich wider in der Bedeutung des persönlichen Gebets, das die Mädchen als Praktik beschreiben, durch die sie Hilfe und Schutz erfahren. Das Mädchen, dessen Vater verstorben ist, beschreibt religiöse Praktiken tröstend; als Bild malt sie einen Sandstrand, auf dem manchmal eine, manchmal zwei Fusspuren zu sehen sind, und erklärt mir, dass dieses Bild dafürstehe, dass Gott sie durch schwere Zeiten trägt. Beide Mädchen berichten von ritualisierten religiösen Praktiken, die sie durchlaufen haben, seitdem sie in der Gruppe leben (Taufe, Kommunion). Religiöse Praktiken werden von den beiden Mädchen entsprechend als Ressource beschrieben, die innen durch das Leben in der Heimgruppe zugänglich gemacht wurde.

Gleichwohl beschreiben die Mädchen auch, dass die Begegnung mit religiösen Praktiken für sie anfänglich eine Fremdheitserfahrung darstellte, der Weihrauch im Gottesdienst wurde als seltsam empfunden und auch das Tischgebet hat zu Beginn verunsichert: „[U]nd ich wusste gar nicht, was man machen muss, als erstes habe ich so gemacht und dann habe ich berichtigt die Hände gefaltet, und dann hab ich irgendwie einfach alle nachgemacht." Auch wird die Begrenzungsfunktion, die mit dem Wissen um (christlich-katholische) religiöse Praktiken einhergeht, deutlich: Ein Mädchen erklärt mir im Interview ausführlich, dass sie Gott nicht malen kann, da dies in den zehn Geboten explizit verboten sei. Der Religionsunterricht wird als langweilige Pflicht erlebt. Die Erfahrung des Pilgerns als Trauerbewältigung wird als hilfreich skizziert, dennoch bleibt das Erleben, dass die Trauer damit nicht bewältigt ist und der Verlust des Vaters auch eine Enttäuschung vom eigentlich als wohlwollend erlebten Gott darstellt, was im Alltag der Heimgruppe wenig thematisierbar ist.

\subsection{Zwischenfazit}

Anschaulich wird in den Beobachtungen und den Interviews mit den Kindern, dass religiöse Praktiken in der untersuchten Einrichtung und in der Heimgruppe diverse Funktionen erfüllen:

Religiöse Praktiken geben Struktur auf verschiedenen Ebenen, sie sind alltagsstrukturierend, v.a. in Bezug auf das gemeinsame Essen, strukturieren den Jahresablauf durch christliche Feste und das Kirchenjahr (Fastenzeit, Adventszeit), teils strukturieren sie für die Kinder auch die Freizeit, wenn diese an Aktivitäten wie Sternsinger und kirchlichen Ferienlagern teilnehmen oder christliche Bücher lesen. Sie sind lebensstrukturierend, wenn Übergänge mit religiösen Praktiken einhergehen, wie Kommunion und Konfirmation, aber auch Pilgern als Trauerverarbeitung. Und sie haben in der Gruppe raumstrukturierende Funktion im weitesten Sinne, wenn ein wichtiger Teil der Raumdekoration auf religiöse Symboliken verweist.

Durch religiöse Praktiken werden existentielle Themen der Kinder angesprochen, sie befördern Gespräche über Tod und Sterben, über Sinnfragen, Werte und Normen.

Durch religiöse Praktiken wird normiert und moralisiert, so wird anhand religiös begründeter Weihnachtspraktiken Konsumkritik geübt, die 
Essenssituation normierend strukturiert, es werden Verhaltensnormen für den Gottesdienstbesuch aufgestellt und es werden über religiöse Praktiken Familiennormalitäten kommuniziert.

5.4 Legitimation religiöser Praktiken durch die Institution und die Mitarbeiter*innen

In den Interviews mit Mitarbeiter*innen der Gruppe und der Abteilungsleitung werden verschiedene Legitimationsmuster für die religiösen Praktiken in der Einrichtung sichtbar, die allerdings auch teils Brüche aufweisen:

Religiöse Praktiken werden dadurch legitimiert, dass

(1) den Kindern ein Interesse an religiösen Themen zugeschrieben wird, das in der Einrichtung in Form religiöser Praktiken aufgegriffen wird. Alle Interviewten betonen, dass die Kinder untereinander viele Gespräche über Religion führen, über existentielle Themen sprechen und religiöse Angebote der Einrichtung wie der örtlichen Kirchengemeinde gerne annehmen. Religiöse Praktiken werden daher als eine Antwort auf ein kindliches Bedürfnis legitimiert.

(2) sie aus der Sicht der Leitungs- und Fachkräfte einen Angebotscharakter aufweisen, keine Verpflichtung. Alle Interviewten betonen, dass es in der Einrichtung wie in der Gruppe viele religiöse Angebote für die Kinder gäbe, aber dass diese immer ein Angebot darstellen, nicht missionarisch seien, nichts Verpflichtendes, und keine Einschränkungen für die Kinder mit sich bringen.

(3) sie aus der Sicht der Einrichtungsleitung wie der Gruppenleitung ausschliesslich Chancen eröffnen und kein Belastungspotential mit sich bringen. Alle Interviewten gehen davon aus, dass es keine Belastungen für die Kinder aus den religiösen Praktiken der Einrichtung geben kann. Begründet wird dies mit dem oben beschriebenen Angebotscharakter. Einschränkungen werden nicht gesehen, auch nicht in Bezug auf die religiös begründete Sexualmoral der Einrichtung für die Jugendlichen (,Liebe und Sex gehören zusammen“), die aus der Sicht der Abteilungsleitung ebenfalls nur Chancen für die Jugendlichen eröffnet.
(4) ihnen generalisierend ein resilienzförderndes Potential zugeschrieben wird. Religion wird in allen Interviews ausschliesslich positiv als Resilienzfaktor für die Kinder konnotiert. Dabei wird der Begriff der Resilienzförderung sehr pauschal und wenig präzise genutzt. Zentrales Anliegen ist es, über Religion ein Zugehörigkeitsgefühl herzustellen (zur Einrichtung, Gruppe, Kirchengemeinde, religiösen Gemeinschaft/Gott im weitesten Sinne). Hier wird von den Interviewten v.a. die Vulnerabilität der Kinder und Jugendlichen betont und deren Bedürfnis nach Zugehörigkeit. Resilienzfördernd sollen religiöse Praktiken entsprechend in ihrer zugehörigkeitsstiftenden und in ihrer trostspendenden Funktion sein.

(5) sie von den Interviewten persönlich als bereichernd erlebt werden; es wird generalisiert, dass sie auch für die Kinder eine Bereicherung darstellen. Der Abteilungsleiter und die Seelsorgebeauftrage setzen sich selbst stark mit ihren persönlichen religiösen Bezügen und Praktiken auseinander. Beide berichten von persönlichen positiven Erfahrungen mit religiösen Praktiken (Gebet, Nächstenliebe) und machen deutlich, dass sie davon ausgehen, dass diese Bereicherung nicht nur subjektiv ist. Die interviewten Erzieher*innen betonen, nicht besonders religiös zu sein, schätzen aber religiöse Angebote für die Mitarbeiter*innen. Der Praktikant dagegen betont, dass seine Tätigkeit in der Gruppe seine persönliche Auseinandersetzung mit der Religion angeregt und dass er selbst angefangen hat, religiöse Praktiken im Alltag, wie das Gebet, umzusetzen.

(6) sie aus der Sicht der Einrichtungsleitung nicht an christlich-katholische Religiosität gebunden sind, sondern einen spirituellen Charakter aufweisen, und damit unabhängig von den institutionalisierten Religionen allen Kindern und Mitarbeiter*innen zugänglich sind. Wichtig ist es der Leitungsebene zu betonen, dass die religiösen Praktiken in der Einrichtung nicht auf den christlichen Glauben verengt sind und dass die religiösen Praktiken keine missionarische Funktion haben, sondern das Anliegen ist, „Kindern [zu] ermöglichen ihre eigene Spiritualität zu finden und diese leben zu können“. Im Reden über diese „Weite“ wird die Einschätzung, dass es für jede*n Anknüpfungspunkte gibt, deutlich: „[E]s ist nichts Ausschließendes da, die Frage, was ist 
dir heilig, das ist so, dass das die Mama sein kann oder irgendwie was anderes, wenn Kinder da ne Antwort drauf geben können, is schon was da." Ein weiter Religionsbegriff und Interreligiosität wird prinzipiell auch von den Gruppenfachkräften bejaht, allerdings gibt es hier einen Bruch: Den Erzieher*innen fällt es schwer, konkrete Beispiele zu benennen. Stattdessen betonen sie, sich mit Praktiken anderer Religionen nicht auszukennen und daher auf christlich-katholische Praktiken zu fokussieren.

(7) der Einrichtungsträger explizit katholisch ist und als solcher anerkannt wird. Die religiösen Überzeugungen und Praktiken verleihen der Einrichtung eine Wertegrundlage für das Handeln aller Mitarbeitenden, wodurch ein Zusammengehörigkeitsgefühl entsteht. Diese Legitimation verweist auf die Hierarchie des Trägers. Sie wird v.a. vor dem Hintergrund einer caritativen Prägung der Einrichtung positiv konnotiert. Ein in den Interviews beschriebenes christliches Menschenbild stellt demnach die Handlungsbasis und einen Konsens - somit auch ein Gefühl des Zusammengehörens - unter den Mitarbeitenden und Kindern dar.

Voraussetzung dafür, dass religiöse Praktiken legitimiert werden können, ist aus der Sicht der interviewten Leitungskräfte, dass das Personal diese mitträgt. Das Personal wird als zentrale Ressource gesehen und soll explizit so ausgewählt werden, dass es die religiösen Praktiken der Einrichtung lebt. Deutlich wird, dass es diesbezüglich in der Realität der Einrichtung Brüche gibt. So diskutieren die interviewten Leitungskräfte die Grenzen, denen sie begegnen - und auch ihre eigene Inkonsistenz, wenn plötzlich religiöse Haltungen der Erzieher*innen mit einer Affinität zu erlebnis- oder tiergestützter Pädagogik gleichgestellt werden:

Es ist aber auch klar, dass man es nicht aufoktroyieren kann, sondern Mitarbeiter, die das selber auch für sich leben können und selber auch für sich als ja Stärkung oder Kraft sehen, dass die das tatsächlich auch im Gruppenalltag leben können, sollen und bitte, bitte dürfen [...] gleichzeitig hat das seine Grenzen, also wenn da jemand ist, der eigentlich selber wenig damit anfangen kann, dann krieg ich das über son Strukturding natürlich nicht rein [...] es gibt die Leute, die sich für Erlebnispädagogik völlig begeistern [...], es gibt welche, die für tiergestützte Pädagogik brennen [...] und ich glaube so gibt es auch Kinder oder Mitarbeiter, die mit, ich sage mal mit dem Thema religionssensible Erziehung Angebote machen, Resilienz[fähigkeit] durch Religionserziehung sehr sehen und leben und das anbieten können.

\section{DISKUSSION UND SCHLUSSFOLGERUN- GEN}

Die Studie muss als Einzelfallstudie gelesen werden, deren Aussagewert für religiöse Praktiken in anderen Einrichtungen der konfessionellen Heimerziehung stark begrenzt ist.

Deutlich wird, dass die Präsenz religiöser Praktiken im Alltag hoch ist, hier zeigt sich die Sichtbarkeit des glaubensgemeinschaftlichen Profils nach aussen und Erfahrbarkeit nach innen, das Ergebnis überschneidet sich folglich mit denen von Ehlke et al. (2017). Während Ehlke et al. jedoch davon ausgehen, dass es für religiöse Praktiken einen Legitimationsdruck gibt, ist an der vorgestellten Untersuchung auffällig, dass die Legitimationen der Fachkräfte für die religiösen Praktiken eher schwach sind: Sie werden nicht pädagogisch, psychologisch oder sozialisatorisch fundiert legitimiert. Die Legitimation beruht überwiegend auf der Anerkennung des Trägers als explizit christlicher Träger in der Region und den persönlichen Überzeugungen der Befragten. Die Rede von der Resilienzförderung ist als kollektives Legitimationsmuster in der Einrichtung zu verstehen, das seine Begründung möglicherweise in der Religionspädagogik hat (vgl. Lorenz 2015), in der Resilienztheorie gibt es keine Anhaltspunkte dafür, dass religiöse Praktiken generell resilienzfördernd sind. In der Studie werden einige Themen deutlich, die diskussionswürdig erscheinen und/oder denen vertieft nachgegangen werden müsste - auch über die vorgestellte Einzelfallstudie hinaus:

(1) Ein Widerspruch findet sich in der Rede vom Angebotscharakter von Religion in den Expert*inneninterviews vs. der beobachteten Präsenz im Alltag. Fraglich ist, ob die Einrichtung ihrem Anspruch, nicht missionarisch zu sein, gerecht werden kann, wenn sie doch möglicherweise auf die Adressat*innen missionarisch - im 
Sinne eines subtilen Zwangs zur Auseinandersetzung mit dem katholischen Glauben - wirkt. Wie es konfessionellen Einrichtungen generell gelingt, zwischen sozialpädagogischen Angeboten und der Kommunikation von Glaubensüberzeugungen auszutarieren, wäre ein interessanter Forschungsbereich.

(2) Von allen erwachsenen Interviewten wird die Vulnerabilität der Kinder und Jugendlichen thematisiert und die Fachkräfte legitimieren religiöse Praktiken auch mit der Vulnerabilität der Kinder. Inwiefern solche paternalistischen Sichtweisen auf Kinder und Jugendliche im Heim in konfessionellen Einrichtungen verbreitet sind und ob sie durch caritative/diakonische Grundhaltungen verstärkt werden, wäre ein weiterer interessanter Diskussions- und Untersuchungsgegenstand.

(3) Religiöse Praktiken durchziehen den Alltag der untersuchten Heimgruppe. Es ist davon auszugehen, dass diese Praktiken eine sozialisatorische Wirkung auf die Kinder und Jugendlichen haben. Wie sich diese Wirkung gestaltet, wäre interessant zu untersuchen; besonders geeignet dafür könnten retrospektive Untersuchungen sein - sowohl bei ehemaligen Heimkindern aus der untersuchten Gruppe als auch aus anderen Gruppen mit vergleichbaren religiösen Praktiken.

(4) Sichtbar wird, dass über religiöse Praktiken in der Gruppe existentielle Themen angesprochen werden, diese aber teils darunter wieder verblassen und gerade nicht in einer aus pädagogischer Perspektive angemessenen, adressat*innengerechten Form behandelt werden. Sehr deutlich zeigt sich dies, wenn die Trauerbewältigung in religiösen Praktiken ritualisiert wird oder Wünsche an die Eltern mit Verweis auf religiös begründete Konsumkritik abgewehrt werden. So können durch die normierende Funktion religiöser Praktiken Bewältigungsmöglichkeiten und Entwicklungschancen beschnitten werden. Möglich ist, dass im Thema religiöse Praktiken in der Heimerziehung die Sozialpädagogik/Soziale Arbeit und die Religion in ihren normierenden Funktionen aufeinandertreffen (vgl. Seelmeyer 2008; Reimer 2017) und dass es gerade in konfessionellen Einrichtungen einer gemeinsamen Reflexion dieser Normierungsfunktion bedürfte, wiederum insbesondere in Bezug auf die sozialisatorischen Wirkungen.

(5) Ein interessantes Spannungsfeld ist der Stellenwert, der der Bedeutung persönlicher religiöser Überzeugungen und Praktiken des Personals von der Leitungsebene zugemessen wird. Die klassische erziehungswissenschaftliche Vorstellung, dass sich das Seelenleben der Erzieher*innen in der Seele des Kindes wiederspiegelt (Kerschensteiner 1955, mit Bezug auf diverse Schriften von Pestalozzi) - und an der jede Erziehung, egal ob familial oder professionell, zu scheitern droht -, könnte sich im Anspruch der Heimleitung spiegeln und hier um eine quasipastorale Komponente aufgeladen sein. Erziehungswissenschaftlich wird die personale Thematik schon lange differenziert und kritisch diskutiert (vgl. Nohl 1982; Colla/Krüger 2013). Auch in der Pastoraltheologie wird der Anspruch an kirchliches Personal kritisch diskutiert (vgl. z.B. Sammet 2018). Was das Zusammenkommen der kirchlichen wie der pädagogischen Ansprüche für Personal(gewinnung) bedeutet, könnte Gegenstand weiterer Untersuchungen sein und knüpft an die quantitative Befragung von Fachkräften und ihren religiösen Praktiken und Überzeugungen von Ehlke et al. (2017) an. Gemeinsam mit Ehlke et al. kann davon ausgegangen werden, dass in der Ausbildung von Fachkräften (v.a. an nichtkonfessionellen Hochschulen) das Thema Religion nicht so behandelt wird, wie es vor dem Hintergrund der Praxisrelevanz geboten wäre.

(6) Ein weiteres Spannungsfeld stellt die Interreligiosität in der Sprache der Interviewten dar vs. die Monoreligiosität in der Praxis der Einrichtung. Interessant wäre es zu untersuchen, ob/wie in anderen konfessionellen Einrichtungen die Idee der Interreligiosität gestaltet wird.

Weit über die skizzierten Themen hinaus stellt sich jugendhilfepolitisch die Frage, wie durchdrungen von religiösen Praktiken der Alltag in der Kinder- und Jugendhilfe sein darf und was die starke Repräsentanz konfessioneller Träger im Bereich der öffentlichen Erziehung für Profession, Ausbildung und Qualitätsdiskurse bedeutet. In der Einzelfallstudie zeigt sich, dass aus der kleinen Untersuchung viele neue Fragen und Themen hervorgehen. Es bedarf ergo einer 
vertiefenden Untersuchung und eines Diskurses, in dem die Sozialpädagogik gefordert ist, Fragen zu Religion und Religionspädagogik zu stellen (vgl. Oelkers 1992) und die De-Thematisierung der religiösen Dimension (Ehlke et al. 2017: 273) im Fachdiskurs aufzugeben.

\section{LITERATUR}

AGJ (2010): Abschlussbericht des Runden Tisches „Heimerziehung in den 50er und 60er Jahren“. Berlin: AGJ.

Behnisch, Michael (2018): Die Organisation des Täglichen - Alltag in der Heimerziehung am Beispiel des Essens. Frankfurt am Main: IGfH.

Clark, Zoe (2018): No Excuses - Über das Verhältnis von Strafen und verzeihenden Care-Beziehungen in der Heimerziehung. In: Diskurs Kindheits- und Jugendforschung, Themenschwerpunk: „Strafe und Disziplinierung von Kindern und Jugendlichen: Ethische Perspektiven",

S. 55-68.

https://doi.org/10.3224/diskurs.v13i1.05

Colla, Herbert/Krüger, Tim (2013): Der pädagogische Bezug - ein Beitrag zum sozialpädagogischen Können. In: Blaha, Kathrin/Meyer, Christine/Colla, Herbert/Müller-Teusler, Stefan (Hrsg.): Die Person als Organon in der Sozialen Arbeit. Erzieherpersönlichkeit und qualifiziertes Handeln. Wiesbaden: VS Verlag, S. 19-53. https://doi.org/10.1007/978-3-531-94216-2_2

Destatis (2018): Statistiken der Kinder- und Jugendhilfe 2016. Erzieherische Hilfe, Eingliederungshilfe für seelisch behinderte junge Menschen, Hilfe für junge Volljährige - Heimerziehung, sonstige betreute Wohnform. Erschienen am 7. Juni 2018. Wiesbaden: Destatis.

Edelbrock, Anke/Schweitzer, Friedrich/Biesinger Albert (Hrsg.) (2010): Wie viele Götter sind im Himmel? Religiöse Differenzwahrnehmung im Kindesalter. Münster: Waxmann.

Ehlke, Carolin/Karic, Senca/Muckelmann, Christoph/Böllert, Karin/Oelkers, Nina/Schröer, Wolfgang (2017): Soziale Dienste und Glaubensgemeinschaften. Eine Analyse regionaler Wohlfahrtserbringung. Weinheim: Beltz. https://doi.org/10.1007/s12592-013$\underline{0136-4}$
Eßer, Florian (2013): Familienkindheit als sozialpädagogische Herstellungsleistung. Ethnographische Betrachtungen zu ,familienähnlichen“ Formen der Heimerziehung. In: Diskurs Kindheits- und Jugendforschung 8 (2), S. 163-176.

Fowler, James (Hrsg.) (1991): Stages of Faith and Religious Development. New York: Crossroad.

Freigang, Werner/Wolf, Klaus (2001): Heimerziehungsprofile. Sozialpädagogische Porträts. Weinheim/Basel: Beltz.

Freudenberger-Lotz, Petra (2012): Theologische Gespräche mit Jugendlichen. Erfahrungen Beispiele - Anleitungen. Stuttgart/München: Calwer/Kösel.

Hart, Tobin (2003): The Secret Spiritual World of Children. Maui: Inner Ocean.

Helfferich, Cornelia (2014): Leitfaden- und Experteninterviews. In: Baur, Nina/Blasius, Jörg (Hrsg.): Handbuch Methoden der empirischen Sozialforschung. Wiesbaden: VS Verlag, S. 559574. https://doi.org/10.1007/978-3-53118939-0_39

Kappeler, Manfred (2018): Mit den Heimkampagnen ging es los. Menschenwürde und Menschenrechte von Kindern und Jugendlichen müssen die Erziehungspraxis in Einrichtungen der Kinder- und Jugendhilfe bestimmen. In: In:

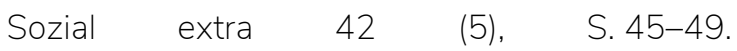
https://doi.org/10.1007/s12054-018-0079-2

Kerschensteiner, Georg (1955): Die Seele des Erziehers. München/Düsseldorf: Verlag von R. Oldenbourg.

Kliche, Helena/Täubig, Vicki (2016): Eine Ethnographie zu Schule in der Heimerziehung zwischen teilnehmender Beobachtung und beobachtender Teilnahme. In: Hitzler, Ronald/Kreher, Simone/Poferl, Angelika/Schröer, Norbert (Hrsg.): Old School - New School? Zur Frage der Optimierung ethnographischer Datengenerierung. Essen: Oldib Verlag, S. 357-366.

Lenz, Karl (1986): Alltagswelten von Jugendlichen. Eine empirische Studie über jugendliche Handlungstypen. Frankfurt am Main: CampusVerlag.

Lorenz, Andreas (2015): Resilienz - wie religiöse Bildung Kinder stärkt. Über die Bedeutung der 
religiösen Bildung in Kindertageseinrichtungen. In: Deutsches Pfarrerblatt 3, S. 154-156.

Maschke, Sabine/Stecher, Ludwig/Coelen, Thomas/Ecarius, Jutta/Gusinde, Frank (2013): Appsolutely smart! Ergebnisse der Studie Jugend.Leben. Bielefeld: WBV. https://doi.org/10.3224/diskurs.v10i1.17706

Merkens, Hans (1992): Teilnehmende Beobachtung: Analyse von Protokollen teilnehmender Beobachter. In Hoffmeyer-Zlotnik, Jürgen H. P. (Hrsg.): Analyse verbaler Daten: über den Umgang mit qualitativen Daten. Opladen: Westdeutscher Verlag, S. 216-247. https://doi.org/10.1007/978-3-322-90092-0_8

Niederberger, Josef Martin (1997): Kinder in Heimen und Pflegefamilien. Fremdplatzierung in Geschichte und Gesellschaft. Bielefeld: Kleine.

Nohl, Herman (1982): Die pädagogische Bewegung in Deutschland und ihre Theorie. Frankfurt am Main: Schulte-Bulmke. https://doi.org/10.30965/9783657768387_12

\section{$\underline{4}$}

Oelkers, Jürgen (1992): Religion: Herausforderung für die Pädagogik. In: Zeitschrift für Pädagogik 38 (2), S. 185-192.

Reimer, Daniela (2017): Normalitätskonstruktionen in Biografien ehemaliger Pflegekinder. Weinheim: Beltz Juventa.

Reimer, Daniela (2018): Die Heimerziehung der Nachkriegszeit war düster. In: Sozial extra 42 (5), S. 50-52. https://doi.org/10.1007/s12054-018$\underline{0080-9}$

Richter, Martina (2013): Die Sichtbarmachung des Familialen. Gesprächspraktiken in der Sozialpädagogischen Familienhilfe. Beltz Juventa.

Rose, Lotte/Adio-Zimmermann, Nora (2018): Ethnografie des Essens in der Heimerziehung. Annäherungen an ein übersehenes, Tagesgeschäft' der stationären Jugendhilfe. In: Aghamiri, Kathrin/Reinecke-Terner, Anja/Streck, Rebekka/Unterkofler, Ursula (Hrsg.): Doing Social Work - Ethnografische Forschung als Theoriebildung. Opladen: Verlag Barbara Budrich. https://doi.org/10.2307/j.ctvddzsh2.12

Sammet, Kornelia (2018): Religiöse Profession. In: Pollack, Detlef/Krech, Volkhard/Müller, Olaf/Hero, Markus (Hrsg.): Handbuch
Religionssoziologie. Wiesbaden: VS Verlag, S. 543-566. https://doi.org/10.1007/978-3531-18924-6_22

Schallberger, Peter/Schwendener, Alfred (2017): Erziehungsanstalt oder Fördersetting? Kinderund Jugendheime in der Schweiz heute. Köln: Herbert von Halem Verlag.

Seelmeyer, Udo (2008): Das Ende der Normalisierung? Soziale Arbeit zwischen Normativität und Normalität. Weinheim, München: Juventa.

Schweitzer, Friedrich (2013): Das Recht des Kindes auf Religion. Gütersloh: Gütersloher Verlagshaus.

Selent, Inga/Strahl, Benjamin/Kohring, Cynthia/Böllert, Karin/Schröer, Wolfgang (2018): Kinder- und Jugendhilfe in muslimischer Trägerschaft. In: Soziale Passagen 10 (2), S. 315-319. https://doi.org/10.1007/s12592-018-0295-4

Schröder, Carsten (2017): Emotionen und professionelles Handeln in der Sozialen Arbeit - Eine Ethnographie der Emotionsarbeit im Handlungsfeld der Heimerziehung. Wiesbaden: Springer VS. https://doi.org/10.1007/s12054-018-0104$\underline{5}$

Schrödter, Mark (2018): Sind Strafen pädagogisch legitimierbar? Das Beispiel der Strafliegestütze. In: Vierteljahrsschrift für wissenschaftliche Pädagogik 94 (2), S. 313-330.

Thiersch, Hans (2014): Lebensweltorientierte Soziale Arbeit. Aufgaben der Praxis im sozialen Wandel. 9. Aufl. Weinheim: Beltz Juventa.

Wensierski, Peter (2006): Schläge im Namen des Herrn. Die verdrängte Geschichte der Heimkinder in der Bundesrepublik. München: Deutsche Verlags-Anstalt (DVA).

Wolf, Klaus (1999): Machtprozesse in der Heimerziehung. Eine qualitative Studie über ein Setting klassischer Heimerziehung. Münster: Votum.

Wolf, Klaus (2005): Pädagogische Mitarbeiterinnen als Potenziale im Lebensfeld von Kindern. Anfragen an professionelle Erziehung (in Lebensgemeinschaften). In: Forum Erziehungshilfen 3, S. 178-182. 


\section{Zur Autorin}

Daniela Reimer ist Dozentin für Kinder- und Jugendhilfe an der Zürcher Hochschule für angewandte Wissenschaften (ZHAW). Sie forscht zu Pflegekinderhilfe, Normalität, Biografie sowie Aufwachsen unter erschwerten Bedingungen.

\section{Kontakt}

Dr. phil. Daniela Reimer

Dozentin für Kinder- und Jugendhilfe

Zürcher Hochschule für angewandte Wissenschaften (ZHAW)

Pfingstweidstrasse 96

8005 Zürich

Tel: +41589348539

E-Mail: daniela.reimer@zhaw.ch

URL: https://www.zhaw.ch/de/ueber-uns/person/remr/ 